Proc. of the 13th Symposium of Magnetic Measurements and Modeling SMMM'2018, Wieliczka, Poland, October 8-10, 2018

\title{
Correcting for FEL Magnetic Field Distortions: The Method of Bilinear Shimming
}

\author{
I. Chaimov*, E. Dyunin and A. Yahalom \\ Department of Electrical and Electronic Engineering, Ariel University, Ariel 40700, Israel
}

\begin{abstract}
A $\mathrm{THz}$ free electron laser is being built in Ariel University. Upon completion it is intended to become a user facility. The free electron laser is designed to emit radiation between 1 and $5 \mathrm{THz}$. It is planned to operate in the super radiance regime. The anticipated output of the free electron laser, is in excess of $150 \mathrm{~kW}$ instantaneous power. An essential part of every free electron laser is a device generating a periodic magnetic field denoted as the wiggler. One of the main requirements of a free electron laser instrument is to achieve nominal magnetic field values with a high accuracy, along the main axis of the free electron laser's permanent magnetic periodic undulator. For practical reasons, mainly due to magnets manufacturing, there are deviations of the magnetic field of the magnets bars which construct the wiggler device in reference to the theoretical magnetic field. In many cases, this deviation reduces the efficiency of the radiation. The magnetic bars magnetization can be treated as a random variable for which we assume to have a normal random distribution having a standard deviation from $5 \%$ to $10 \%$ (depending on the quality of magnet's manufacturing). Our main purpose is to optimize the magnetic field by adding small dipole-like magnets which enable corrections for the noise in practical magnetic fields. This enables us to minimize the unwanted field noise and achieve a more optimal wiggler device.
\end{abstract}

DOI: 10.12693/APhysPolA.136.745

PACS/topics: 03.50.De, 07.55.--w, 07.55.Db, 07.57.Pt, 41.85.Lc

\section{Introduction}

One of the main requirements of a free electron laser (FEL) instrument is to achieve nominal magnetic field values with a high accuracy, along the main axis of the FEL permanent magnetic periodic undulator known as the wiggler which usually takes the form of the Halbach [1] configuration. It is obvious that every sub-system which creates the FEL system affects the overall system accuracy, however, the wiggler is a key device whose faulty construction can significantly deteriorate the overall performance of the entire FEL system. For practical reasons, mainly due to magnets manufacturing, there are deviations of the magnetic field of the magnets bars which construct the wiggler in reference to the theoretical magnetic field. In many cases, this deviation reduces the efficiency of the radiation extraction, reducing the delivered power and energy extracted from the device and hence, giving rise to undesirable heat absorbed at the device, which in some cases can cause a de-magnetization affect at the magnet bars. The magnetic bars magnetization could be treated as a random variable for which we assume to have a normal random distribution having a standard deviation from $5 \%$ to $10 \%$ (depending on the quality of the magnet's manufacturing). Our main purpose is to optimize the magnetic field by adding small magnets, which enable corrections for the noise in practical magnetic fields. This enables us to minimize the unwanted field noise and achieve an optimal wiggler device. We take advantage of a simple

*corresponding author; e-mail: itzik.chaimov@outlook.com analytic formula that exists for the magnetic field due to small magnetic elements, which is the dipole formula that depends linearly on the strength of the dipole.

Various methods have been used to improve wiggler's imperfection, for example, adjustment of the magnetic field using a mathematical programming method for an undulator [7], synthesized 3D field simulation of trajectories and sorting of lateral focusing magnets [5], magnet sorting using simulated annealing [6], new system for wiggler fabrication and testing [4].

This article begins with an introduction to the wiggler (Halbach) principle characteristics which includes its physical composition and associated theoretical magnetic field. This is compared with the practical magnetic field distribution. It continuous with the discussion of the primary optimization algorithm which assumes a continuous range of dipole values. This theoretical assumption is later dropped as we consider the case of discrete valued dipoles better suited for engineering practical requirements. The article concludes with a summary of the various results.

\section{Wiggler principle characteristics}

The following subsection discusses, briefly, the common characteristics of a practical noisy linear (Halbach) wiggler.

A wiggler is a device consisting of a collection of magnets which are arranged in two rows in order to produce a periodic magnetic field $[1,2]$.

As a first step of this research we simulated the magnetic field using the CST EM magnetostatic solver, which is a commercial software. Figure 1 demonstrates the wiggler structure (and also depicts the choice of axis). 
(a)
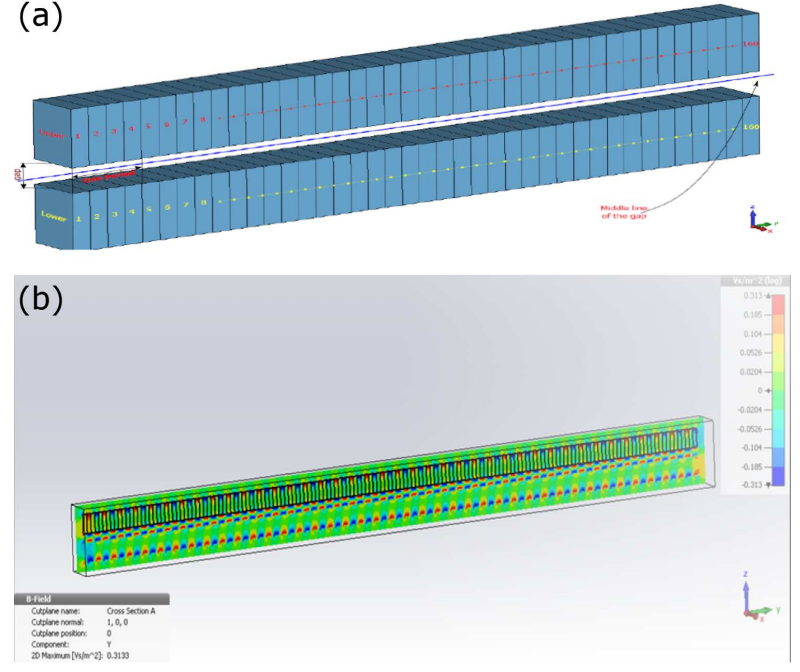

Fig. 1. Field distribution in a wiggler structure. (a) Geometry of the wiggler used in the CST simulation.

(b) Simulated magnetic field at a cross-sectional plan.

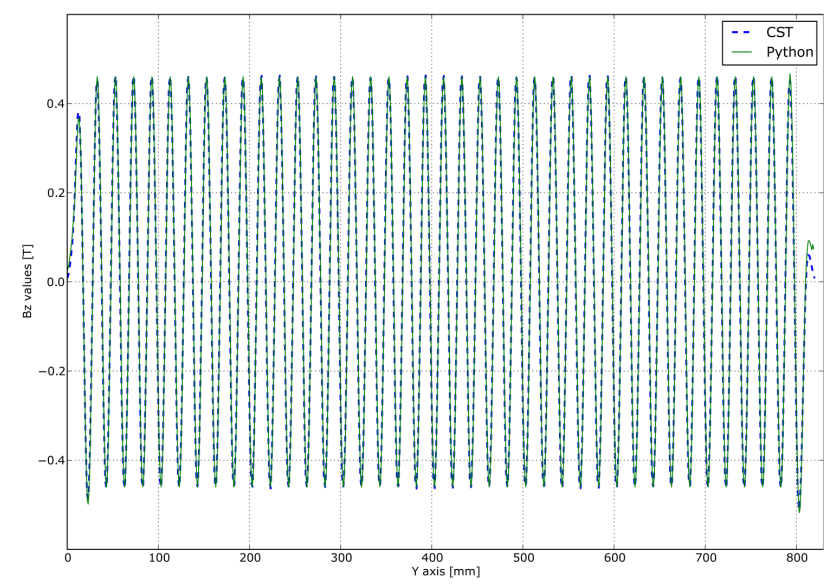

Fig. 2. Magnetic field distribution along the wiggler symmetry axis: comparison between CST simulation and Python calculation.

The results of simulating the magnetic fields of the wiggler along the device symmetry axis which is the required electron path is given in Fig. 2. The field is calculated starting from the wiggler jaws at $y=0$ till the wiggler end at $y=800 \mathrm{~mm}$.

Figure 2 also shows a comparison between a CST simulation and an analytical calculation of the magnetic field implemented using the Python programming language. The Python developed code uses explicit expressions given by [3] for the magnetic field of a uniform block magnet. As can be seen, the two distinct methods give almost identical results which increases our confidence in both the simulation and analytical calculation.

In practice, there are deviations between the magnetic field generated by each bar magnet due to deficiencies in the process of magnet manufacturing. This can be

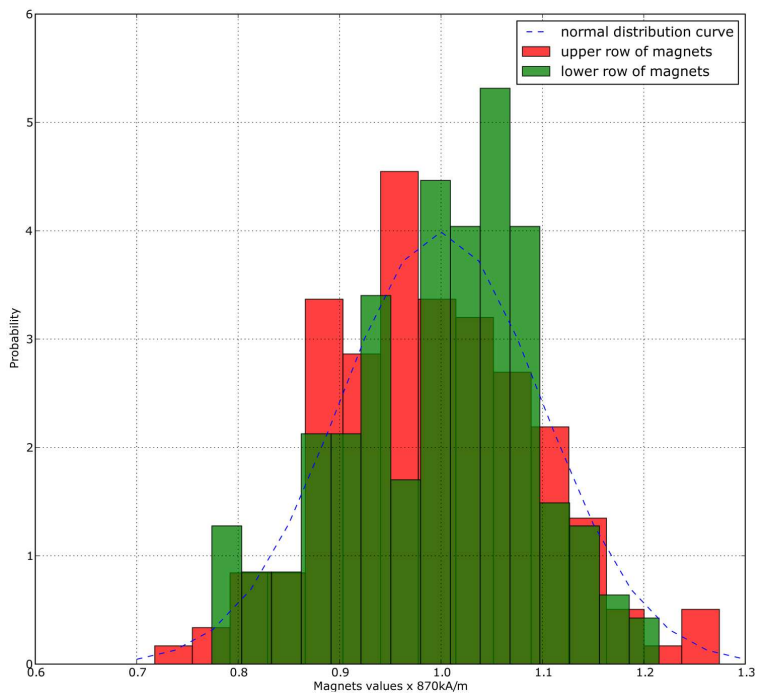

Fig. 3. Two histograms plots of 160 magnetization random values (each).

Design case summary

TABLE I

\begin{tabular}{l|c}
\hline \hline total number of bar magnets & 320 \\
bar magnets in a row & 160 \\
number of rows & 2 \\
total length [mm] & 800 \\
period length [mm] & 20 \\
number of periods & 40 \\
magnet bar dimensions: & \\
$\quad X$ [mm] & 15 \\
$Y$ [mm] & 5 \\
$Z$ [mm] & 20 \\
magnetization [kA/m] & 870 \\
noise std. deviation [\%] & 10 \\
peak magnetic field value [T] & 0.48 \\
wiggler's gap [mm] & 8.71
\end{tabular}

treated as a noise with a normal distribution. We demonstrate here the case of a $10 \%$ standard deviation. Figure 3 shows the (normal) random distribution for bar magnets in both rows (upper and lower) of the manufactured magnets of the wiggler device. The design parameters of the wiggler device are summarized in Table I.

\section{Applying optimization algorithm}

\subsection{Theoretical (continuous dipole values) case}

To achieve an optimized magnetic field, we spread small magnets bars along the $Y$-axis of the wiggler device with magnetizations directed in the $Z$-direction.

The developed algorithm gives an upper bound for the quality of the magnetic field due to dipole magnets located along the said device. 
The magnetic field $\boldsymbol{B}$ generated by a single dipole of strength $m$ oriented in the $Z$-direction at a distance $\boldsymbol{X}$ from the dipole is [8]:

$$
\boldsymbol{B}(\boldsymbol{X})=\frac{\mu_{0}}{4 \pi} m \frac{3 \hat{n}(\hat{n} \cdot \hat{z})-\hat{z}}{|\boldsymbol{X}|^{3}}=\boldsymbol{B}_{n}^{k} .
$$

Here $\hat{n}=\frac{\boldsymbol{X}}{|\boldsymbol{X}|}, \hat{z}$ is a unit vector in the $Z$-direction, and $\mu_{0}$ is the magnetic permeability of the vacuum. The magnetic field $\boldsymbol{B}$ generated by a single dipole of strength $m$ located at position $\boldsymbol{x}_{k}$ at position $\boldsymbol{x}_{n}$ is thus

$$
\boldsymbol{B}\left(\boldsymbol{X}_{n k}\right)=m \boldsymbol{B}_{n}^{k}, \quad \boldsymbol{X}_{n k} \equiv \boldsymbol{x}_{n}-\boldsymbol{x}_{k}
$$

where

$$
\boldsymbol{B}_{n}^{k}=\frac{\mu_{0}}{4 \pi} \frac{3 \hat{n}_{n k}\left(\hat{n}_{n k} \cdot \hat{z}\right)-\hat{z}}{\left|\boldsymbol{X}_{n k}\right|^{3}} .
$$

We denote the actual noisy distribution of the magnetic field emerging from the wiggler structure at the position $\boldsymbol{x}_{n}$ by $\boldsymbol{B}_{n}^{a}$. The required magnetic field is denoted as $\boldsymbol{B}_{n}^{r}$. The difference between the actual and required field at point $n$ is given by

$$
\Delta \boldsymbol{B}_{n}=\boldsymbol{B}_{n}^{a}-\boldsymbol{B}_{n}^{r}
$$

The target function $T_{f}$ with $m_{k}$ as the vector variable is thus defined as:

$$
T_{f}(\boldsymbol{m})=\frac{1}{2} \sum_{n}\left(\Delta \boldsymbol{B}_{n}-\sum_{k} m_{k} \boldsymbol{B}_{n}^{k}\right)^{2},
$$

where the summation is performed over all the points $n$ where the field is measured. In an ideal situation the dipole field will cancel out the difference between the required and actual field at every spatial point and $T_{f}$ will vanish. However, in other cases there is a minimum value for $T_{f}$ as will be shown below. Equation (5) can be written as a bilinear form

$$
T_{f}(\boldsymbol{m})=C+\boldsymbol{m} \cdot \boldsymbol{F}+\frac{1}{2} \boldsymbol{m} \hat{L} \cdot \boldsymbol{m},
$$

where

$$
C=\frac{1}{2} \sum_{n} \Delta \boldsymbol{B}_{n}^{2}
$$

is a real number.

$$
F_{k}=\sum_{n} \boldsymbol{B}_{n}^{k} \Delta \boldsymbol{B}_{n}
$$

is a vector whose dimension is equal to the number of dipoles.

$$
\hat{L}_{k l}=\sum_{n} \boldsymbol{B}_{n}^{k} \boldsymbol{B}_{n}^{l}
$$

is a matrix where both dimensions are equal to the number of dipoles. The minimum of this target function is obtained as follows:

$$
0=\frac{\partial T_{f}(\boldsymbol{m})}{\partial m_{i}}=F_{i}+\sum_{l} \hat{L}_{i l} m_{l}=\boldsymbol{F}+\hat{L} \boldsymbol{m}
$$

Hence we obtain the optimal dipole values

$$
\boldsymbol{m}=-\hat{L}^{-1} \boldsymbol{F} \text {. }
$$

The minimum value of the target function

$$
T_{f \min }=C-\frac{1}{2} \boldsymbol{F} \hat{L}^{-1} \boldsymbol{F}
$$

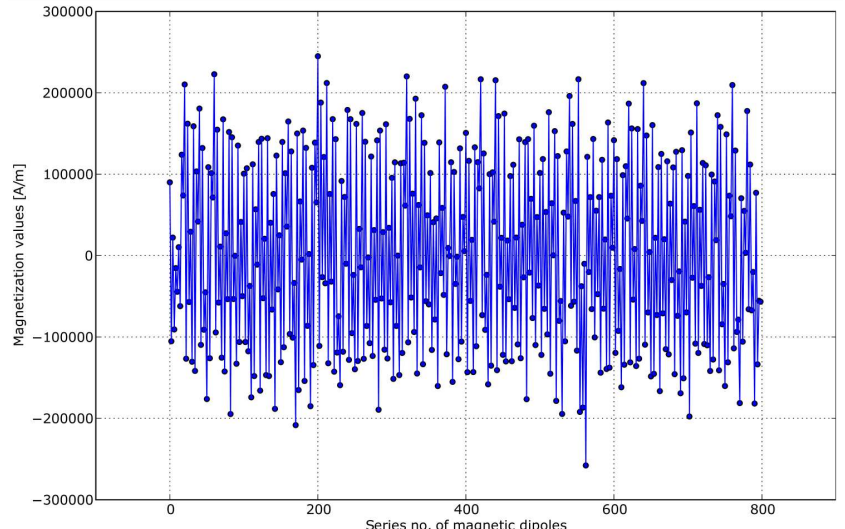

Fig. 4. Magnetization values for 160 dipoles.

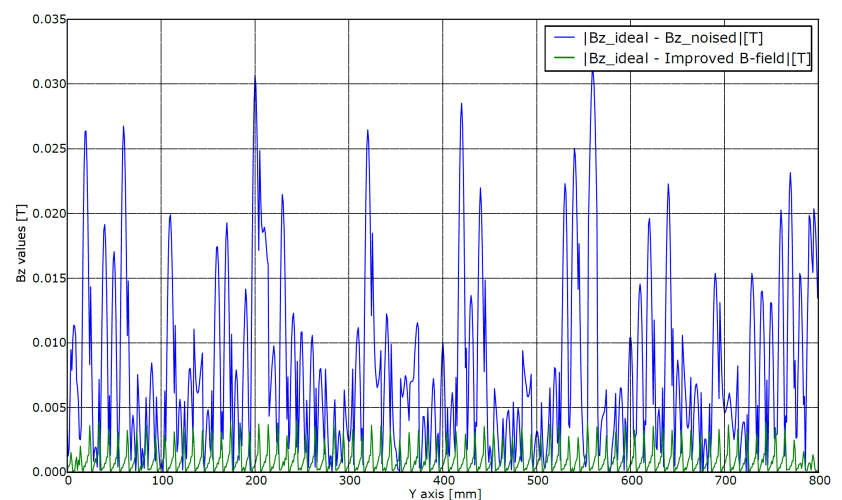

Fig. 5. Deviation from the required magnetic field values before and after correction.

In practice not every dipole value is achievable, hence one cannot obtain $T_{f} \min$. However, using realizable discretization techniques we obtained considerable improvement in the quality of the magnetic field.

The obtained $\boldsymbol{m}$ values of the algorithm are depicted in Fig. 4. A comparison between the deviation of the actual (noisy) field before and after correction using the $\boldsymbol{m}$ values of Eq. (1) is depicted in Fig. 5. The target function Eq. (6) of the uncorrected scenario $(\boldsymbol{m}=0)$ equals 0.0326 while the target function value after applying the correcting algorithm achieved a significant improvement and is equal to 0.00075113 . Also, it is useful to quantify the peak-to-peak values of the deviation given at the uncorrected case which is equal to $0.06167 \mathrm{~T}$ and the corrected case peak-to-peak which equals 0.007893 . Finally, we also calculate the standard deviation. Before the correction we have 0.1 (10\% starting case), and after correction with continuous dipoles we obtain improved standard deviation of 0.00138 (which is 1.38\%).

\subsection{Practical (discrete dipole values) case}

The previous section presented the main algorithm which solves the problem of the magnetic deviations. 
The results we obtained are continuous and impractical with respect to engineering feasible implementation. Here we will extend the formulation and algorithm for which we can obtain the final practical solution to the magnetic field deviation problem.

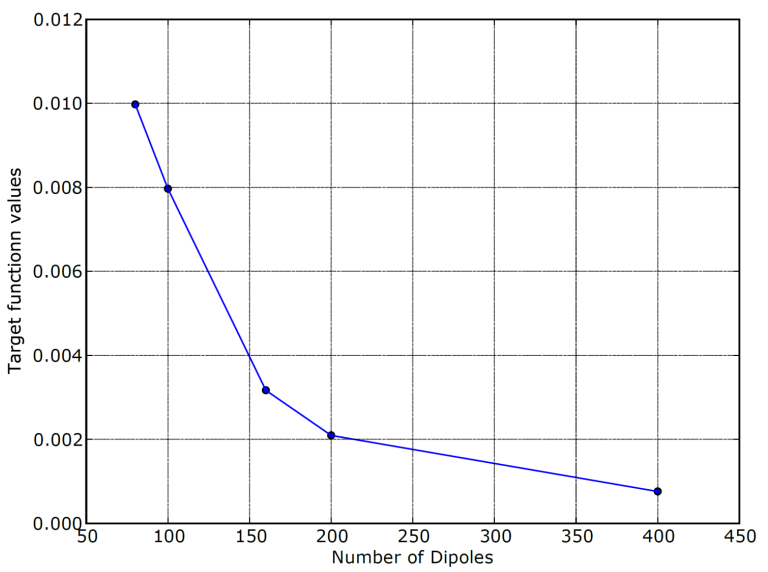

Fig. 6. Target function values of the five selected cases.

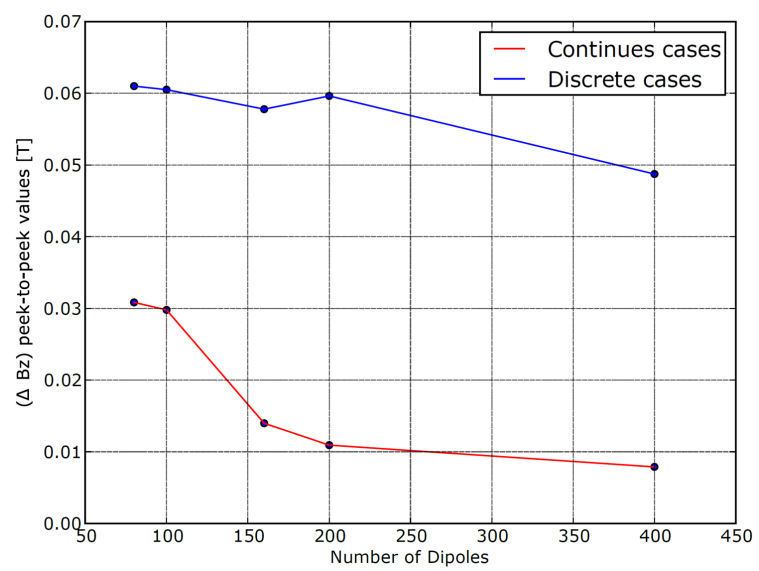

Fig. 7. Peak-to-peak values: comparison of continuous and discrete cases.

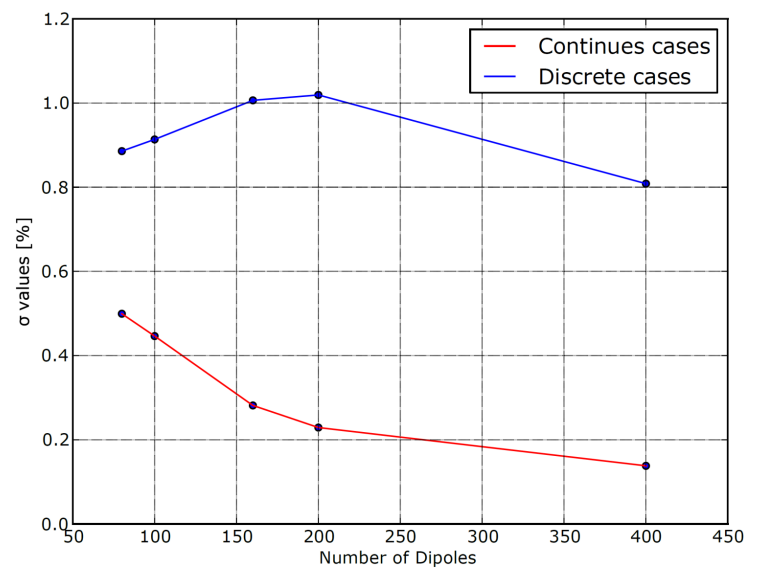

Fig. 8. Standard deviation values: comparison of continuous and discrete cases).
While the previous section finds the optimized magnetic dipole moments array using the inverse-matrix of Eq. (11), the main novelty in this section is to find the discrete values of magnetic dipole moments from the already obtained continuous $\boldsymbol{m}$ values. Let us denote the discrete-sized $\boldsymbol{m}$ values as

$$
\boldsymbol{m}_{d}=\operatorname{discrete}\{\boldsymbol{m}\} \text {. }
$$

For those values the target function obtained is

$$
T_{f}\left(\boldsymbol{m}_{d}\right)=\frac{1}{2} \sum_{n}\left(\Delta \boldsymbol{B}_{n}-\sum_{k} m_{d k} \boldsymbol{B}_{n}^{k}\right)^{2} .
$$

Please note that the following inequality should be satisfied when obtaining the new target function values:

$T_{f \text { min:continuous }} \leq T_{f \text { min:discrete }}$.

The inequality means that target function value at the discrete case are at the best case equal to the target function value obtained at the best continuous case. The target function yielded the optimal values depicted in Fig. 6 which demonstrate the convergence of the solution as required.

Figures 7 and 8 depict the peak-to-peak and standard deviations values for five calculated cases of dipole spacing (or in other words number of total dipoles): for every $10 \mathrm{~mm}$ (80 dipoles), $8 \mathrm{~mm}$ (100), $5 \mathrm{~mm}$ (160), $4 \mathrm{~mm}$ (200), and $2 \mathrm{~mm}$ (400) spacing.

\section{Conclusion}

The quality of the wiggler structure magnetization correction values depends on few factors. The most important are the number of correcting elements, resolution of discrete values, and finally choosing the correct finetune discrete algorithm to fit the discrete values correctly. We saw that there are some cases where we expected to see improvement but we saw deterioration of the quality in the discrete case. This could be explained by the fact that the discrete values are not correctly chosen. Based on our work we recommend a wiggler correction scheme with 400 dipole elements as can be deduced from the last two graphs.

\section{References}

[1] K. Halbach, Nucl. Instrum. Methods 169, 1 (1980).

[2] K. Miyata, K. Ohashi, IEEE Transl. J. Magn. Japan 7, 259 (1992).

[3] H.S. Marks, M. Volshonok, E. Dyunin, A. Gover, Y. Lasser, R. Shershevski, A. Yahalom, Nucl. Instrum. Methods Phys. Res. A 9, 15 (2001).

[4] B. Faatz, J. Pfluger, TESLA FEL-Report 1999-01, Deutsches Elektronen Synchroton DESY, 1999.

[5] R.W. Warren, C.J. Elliot, in: Proc. Adriatico Research Conf. on Undulator Magnets for Synchrotron Radiation and FEL, Trieste 1987, World Sci., 1988.

[6] N. Balal, E. Magori, A. Yahalom, Acta Phys. Pol. A 128, 259 (2015).

[7] R. Engel-Herbert, T. Hesjedal, J. Appl. Phys. 97, 2005.

[8] J.D. Jackson, Classical Electrodynamics, 3rd ed., Wiley, New York 1999. 